\title{
Methods for assessing the severity of perinatal asphyxia and early prognostic tools in neonates with hypoxic-ischemic encephalopathy treated with therapeutic hypothermia
}

\author{
Wojciech Walas ${ }^{1, A-F}$, Maria Wilińska ${ }^{2, A, B, F}$, Monika Bekiesińska-Figatowska ${ }^{3, A, B, F}$, Zenon Halaba ${ }^{4, A, B, F}$, Robert Śmigiele ${ }^{5, A, B, E, F}$ \\ ${ }^{1}$ Pediatric and Neonatal Intensive Care Unit, University Hospital in Opole, Poland \\ 2 Department of Neonatology, Centre of Postgraduate Medical Education, Warszawa, Poland \\ ${ }^{3}$ Department of Diagnostic Imaging, Institute of Mother and Child, Warszawa, Poland \\ ${ }^{4}$ Department of Pediatrics, Institute of Medical Sciences, University of Opole, Poland \\ ${ }^{5}$ Department of Pediatrics, Division of Propaedeutic Pediatrics and Rare Disorders, Wroclaw Medical University, Poland \\ A - research concept and design; B - collection and/or assembly of data; $C$ - data analysis and interpretation; \\ $\mathrm{D}$ - writing the article; $\mathrm{E}$ - critical revision of the article; $\mathrm{F}$ - final approval of the article
}

Address for correspondence

Wojciech Walas

E-mail:wojciechwalas@wp.pl

Funding sources

This research was partially carried out within framework of the National Science Centre (NCN), Poland, project NNo. UMO-2018/29/B/ST8/01490.

Conflict of interest

None declared

Received on April 28, 2020

Reviewed on May 7, 2020

Accepted on June 22, 2020

Published online on August 21, 2020

\begin{abstract}
Despite the progress in perinatal care, perinatal asphyxia (PA) remains a significant problem in neonatology. The development of therapeutic hypothermia (TH) has improved the prognosis, but it still remains uncertain in hypoxic neonates. The evaluation of the severity of ischemia/hypoxia after birth is crucial to the choice of treatment, and with accurate long-term prognosis, appropriate further patient care can be planned. This article presents various methods for the preliminary assessment of brain damage and prognosis in newborns with PA treated with TH. The importance of assessing the neurological condition and the usefulness of laboratory and electrophysiological testing and imaging are discussed. New methods are also noted, which are at the stage of clinical trials. A combination of the prognostic tests presented in this article can provide greater prognostic accuracy for predicting long-term neurological outcomes in infants with hypoxic-ischemic encephalopathy (HIE) undergoing TH than either of these tests independently. Acknowledging the limitations of individual tools in certain clinical situations and the integration of the information available from multiple biomarkers may help improve the accuracy of prognostication.
\end{abstract}

Key words: perinatal asphyxia, neonate, prediction factors, therapeutic hypothermia, hypoxic-ischemic encephalopathy

Cite as

Walas W, Wilińska M, Bekiesińska-Figatowska M, Halaba Z, Śmigiel R. Methods for assessing the severity of perinatal asphyxia and early prognostic tools in neonates with hypoxic-ischemic encephalopathy treated with therapeutic hypothermia. Adv Clin Exp Med. 2020;29(8):1011-1016. doi:10.17219/acem/124437

DOI

10.17219/acem/124437

Copyright

Copyright by Author(s)

This is an article distributed under the terms of the

Creative Commons Attribution 3.0 Unported (CC BY 3.0)

(https://creativecommons.org/licenses/by/3.0/) 


\section{Introduction}

Despite recent advances in perinatal care, neonatal hypoxic-ischemic encephalopathy (HIE) is one of the most common causes of severe neurological deficit in children, present in approx. 15 out of 10,000 live births. ${ }^{1}$ According to the World Health Organization (WHO), perinatal asphyxia (PA) represents the $3^{\text {rd }}$ most common cause of neonatal death (23\%). This means that all over the world, almost 600,000 newborns die every year, and at least as many develop severe complications such as epilepsy, cerebral palsy and developmental delay due to acute perinatal sentinel events. ${ }^{2}$ Therapeutic hypothermia (TH) is a method of treatment that has proven effective in protecting the brain against the effects of ischemia/hypoxia in neonates after PA; it is recommended in both term and near-term newborns. ${ }^{3,4}$ The qualification criteria and contraindications for $\mathrm{TH}$ are adjusted as knowledge progresses and they are included in recommendations and standards for the treatment of newborns. In this age group, 2 methods of cooling are available: selective head hypothermia and whole body hypothermia. Although the incidence rate of HIE is known and TH is increasingly available, no accurate data on the frequency of this treatment are available.

The mechanism for such neurological protection is thought to be multifactorial, including limitation of postarrest endothelial dysfunction, decreased free radical release and blunting of the post-reperfusion inflammatory cascade. Early evaluation of the degree of brain damage in newborns after PA is of great importance in determining the appropriate management and prognosis. This assessment is based on a complex analysis of different factors.

The aim of this article is to present the methods used to estimate the severity of PA and the prognostic tools for neonates with HIE treated with $\mathrm{TH}$.

\section{Clinical assessment of neurological status}

The neurological status at birth and during treatment is routinely assessed in neonatal wards. Assessing a newborn's neurological status after PA is usually difficult due to the serious overall condition and the need to use drugs which affect the brain. The use of scales makes it possible to objectify the evaluation and compare results between different patients.

The Apgar scale is primarily used to assess the condition of a newborn at birth and is one of the basic criteria for pre-qualification for $\mathrm{TH}$ treatment. It is also useful as a prognostic tool, though the results of studies are ambiguous. Laptook et al. showed that the Apgar score at 10 min provides useful prognostic data for infants with HIE. They noticed that death or moderate/severe disability is common, but not uniform, with Apgar scores $<3 .{ }^{5}$ Shah et al. found that $1 / 3$ of infants with a 10-min Apgar score of 0 who survived to reach the neonatal intensive care unit had normal scores on formal developmental assessments. ${ }^{6}$

The Sarnat scale estimates the severity of neurological disorders in newborns after PA. It distinguishes 3 stages of HIE: stage 1 - mild encephalopathy associated with hyperalertness, sympathetic overdrive and a normal electroencephalogram (EEG); stage 2 - moderate encephalopathy marked by obtundation, hypotonia, multifocal seizures, and an EEG showing periodic or continuous delta activity; and stage 3 - severe encephalopathy in which infants are stuporous and flaccid with an isoelectric or periodic EEG. This scale is one of the factors which is widely used in qualifying a newborn for TH treatment, but it is also a prognostic tool. The creators of the scale noted that infants who did not enter stage 3 and who had signs of stage 2 for fewer than 5 days had developed normally on follow-up, but persistence of stage 2 for a week or failure of the EEG to normalize predicted later neurological impairment or death. ${ }^{7}$ Short-term neurological improvement expressed by the Sarnat scale predicts a neurodevelopmental outcome at $18-24$ months. $^{8}$

The Thompson scale is also suitable for assessing the neurological status of neonates after PA. It takes into account 9 parameters; the score ranges from $0-22$ points and is directly proportional to the severity of the neurological condition. This scale is also helpful in determining the prognosis for neurological development. Thompson et al. noted that children with a maximum of 10 points developed properly in the $1^{\text {st }}$ year of life, while $65 \%$ of those with more than 10 points and $92 \%$ with more than 15 points developed incorrectly. ${ }^{9}$ Mendler et al. demonstrated the usefulness of this scale as a prognostic tool. ${ }^{10}$

\section{Laboratory tests}

Among the laboratory tests, acid-base balance holds a special place because metabolic acidosis is the $2^{\text {nd }}$ prequalification criterion for TH treatment, next to the Apgar assessment. The consensus is that the severity of lactacidemia reflects the degree of fetal hypoxia-ischemia, but a single lactate measurement gives no definitive information regarding the duration of asphyxia. Metabolic acidosis also has a prognostic value. Worsening metabolic acidosis at birth correlated with severe brain injury in neonates who were treated with TH. ${ }^{11}$ Moreover, higher serum level of lactate following $\mathrm{TH}$ and abnormal results of brain magnetic resonance imaging (MRI) are associated with a poor neurodevelopmental outcome. ${ }^{12}$ Results obtained by other authors are similar. Also, the meta-analysis carried out by Malin et al. showed that low arterial cord $\mathrm{pH}$ was significantly associated with neonatal mortality, HIE, intraventricular hemorrhage, or periventricular leukomalacia. ${ }^{13}$

Because PA affects the whole body, it is understandable that it causes an increase in the activity of enzymes which are markers of damage to various organs. Liver enzymes, 
myocardial injury biomarkers and other enzymes are often measured in newborns with HIE, being widely available and relatively cheap. Muniraman et al. demonstrated a relationship between the degree of hypoxia and transaminases (aspartate transaminase (AST) and alanine transaminase (ALT)), alkaline phosphatase and ammonia levels. ${ }^{14}$ Research conducted by Montaldo et al. showed that early cardiac troponin I (CT-1) concentration correlates with the severity of HIE and with development at 18 months. ${ }^{15}$ It is suggested to use CT-1, myoglobin and creatine kinase-Mb as new biomarkers for diagnosis of neonatal HIE. ${ }^{16}$ Changes in lactate dehydrogenase are associated with central gray matter lesions and may be a useful biomarker for predicting future neurodevelopmental prognosis in infants with HIE. ${ }^{17}$ Although the hypoxia markers mentioned above have been studied many times and are quite widely used in clinical practice, there are currently no consistent data to establish thresholds corresponding to HIE severity.

A consistent finding across a number of studies is the upregulation of the innate immune system, as evidenced by the increased levels of cytokines in newborns with HIE. Levels of inflammatory interleukins (ILs; IL-1ß, IL-2, IL-4, IL-6, IL-8, IL-10, IL-13, and IL-10), monocyte chemotactic protein-1 (MCP-1) and tumor necrosis factor $\alpha$ (TNF- $\alpha$ ) increase significantly in newborns with HIE and correlate with both the severity of HIE and outcomes. ${ }^{18}$ Attention was also paid to the usefulness of determining the levels of specific biomarkers targeting the so-called "neurovascular unit", which may be helpful in assessing the severity of HIE and prognosis, but which are currently at the stage of clinical trials, are usually expensive and are difficult to obtain. Calcium-binding proteins and other brain biomarkers have been taken into account. Roka et al. and Giuseppe et al. noted that serum S100B protein and neuronspecific enolase (NSE) levels are elevated in newborns with HIE. ${ }^{19,20}$ Moreover, it was also shown that serum S100B and NSE levels in babies with HIE are associated with neurodevelopmental disorders at 15 months. ${ }^{21}$ Ennen et al. noted that serum glial fibrillary acidic protein levels during the $1^{\text {st }}$ week of life were elevated in neonates with HIE and were predictive of brain injury on MRI. ${ }^{22}$ Florio et al. have shown that newborns with HIE have elevated serum levels of activin A and concluded that this parameter is also taken into account as a prognostic tool. ${ }^{23}$ Douglas-Escobar et al. showed that ubiquitin C-terminal hydrolase L1 levels have the potential to predict long-term neurological disorder in these patients. ${ }^{24} \mathrm{Lv}$ et al. noted the predictive value of serum tau protein level for neurodevelopmental outcome in neonates with HIE. ${ }^{25}$

There have been a few publications on the basis of which it can be assumed that other markers may prove useful in assessing HIE severity and predicting prognosis. The following markers have been considered: plasma neurofilament light protein, interferon, sercetoneurin, osteopontin, monocyte chemotactic protein-1, macrophage inflammatory protein 1a, vascular endothelial growth factor (VEGF), leptin, adiponectin, and erythropoietin. ${ }^{26-31}$ The metabolic products of HIE reveal early biomarkers of injury which deprive the neonatal brain of oxygen and glucose, and which trigger a biochemical cascade of many adverse reactions. The details and range of metabolic change occurring in response to HIE remains unclear, but research on the relationship between the severity of HIE and metabolomic profile analysis in umbilical cord blood, serum and urine (mainly amino acid, acylcarnitine and organic acid profiles) is promising and suggest they might be used as a new prognostic tool and treatment targets, but these studies are at the experimental stage. Moreover, the laboratory equipment for metabolome assays is not very accessible. ${ }^{32,33}$

\section{Electrophysiological tests}

Among electrophysiological tests, amplitude-integrated EEG (aEEG) plays a special role, because it is one of the qualification factors for TH treatment and it is routinely monitored during such treatment. It requires 3 electrodes to be placed on the head of the newborn; it is quite easy to perform and the recording is interpreted in comparison to several patterns. Continuous monitoring of aEEG allows the bioelectrical function of the brain and its trend to be estimated. It is also very useful in detecting subclinical seizures. Its usefulness in the prognosis of newborns after PA has also been demonstrated. Liu et al. noted that aEEG reflects the degree of the lesion as well as the long-term prognosis of newborns with HIE. ${ }^{34}$ The usefulness of aEEG in the prognosis of newborns after PA has been confirmed in research conducted by other authors. ${ }^{35-37}$ Also, from the systematic literature review carried out by Del Río, it follows that aEEG background activity, recorded during the first $72 \mathrm{~h}$ of life, has a strong predictive value in infants with HIE treated with $\mathrm{TH}$ or not. In another meta-analysis, the authors concluded that a persistently abnormal aEEG at $48 \mathrm{~h}$ or later is associated with an adverse neurodevelopmental outcome. ${ }^{38,39}$ The implementation and especially the interpretation of the classic, multi-channel EEG is more difficult, but provides additional information on the degree of brain damage and prognosis. It has been noted that electrographic seizure burden is associated with severity of brain injury on MRI in newborns with HIE undergoing $\mathrm{TH}^{40}$ Other authors have demonstrated that burst suppression, low voltage and flat traces in the EEG of term neonates with HIE most accurately predict the long-term neurodevelopmental outcome and that excessive EEG discontinuity is associated with increased cerebral tissue injury on MRI and is predictive of abnormal neurodevelopmental outcome in infants treated with $\mathrm{TH} .{ }^{41,42}$ Weeke et al. noted that severely abnormal EEG background activity at $36 \mathrm{~h}$ and $48 \mathrm{~h}$ of life was associated with severe injury on MRI and abnormal neurodevelopmental outcomes. ${ }^{43}$ A meta-analysis performed by Han et al. found that EEG 
background activity is predictive of long-term neurological outcome in TH-treated neonates with HIE, and that burst suppression, low voltage and flat trace are potential predictors of death or neurodevelopmental impairment. ${ }^{44}$ Another systematic review and meta-analysis confirms the immense usefulness of EEG as a prognostic tool. ${ }^{45}$

The brain injury after PA affects various areas of the brain and may also include the brainstem. Brainstem evoked potentials (BEPs) are used to diagnose various types of brain damage. Some authors note the usefulness of the BEPs in predicting prognosis in newborns with HIE, while others have reported that a bilateral absence of cortical somatosensory evoked potentials (SSEPs) predicts moderate/severe MRI pattern of injury in newborns treated with TH. ${ }^{46}$ Cainelli et al. considered that among BEPs, visual evoked potentials (VEP) constitute the single best neurophysiological prognostic marker, but the combination of neurophysiological tests is more valuable. ${ }^{47}$

\section{Neuroimaging}

Neuroimaging plays a special role in determining the prognosis of newborns with HIE. Cranial ultrasound (CUS) is a readily available, inexpensive bedside examination that is routinely performed on children with HIE, but its prognostic value is limited. It has several limitations, such as low sensitivity for detecting cortical, cerebellar and brainstem lesions, marked inter-observer variability, and operator dependency. Edema dominates in early brain ultrasound imaging in newborns after PA, and assessment of its echostructure is difficult and subjective. Cranial Doppler ultrasonography is a method that objectively assesses cerebral arterial blood flow and resistance. Measurements using the Doppler technique are usually taken in the middle cerebral artery. It was noted that $\mathrm{TH}$ reduced the importance of measuring resistance in cerebral vessels; nevertheless, Gerner et al. found that pre- and post-cooling transfontanellar duplex brain sonography resistive index (RI) values may be a useful prognostic tool, in conjunction with other clinical information for neonates with HIE. ${ }^{48}$ Likewise, Annink et al. developed and validated a CUS scoring system that takes into account the RI and the sum of deep grey matter and white matter involvement, and noted that it is associated with neurodevelopmental outcome in these patients. ${ }^{49}$

The importance of computed tomography $(\mathrm{CT})$ in diagnosing HIE and predicting prognosis has diminished considerably with the popularization of MRI. The CT is less sensitive and less specific than MRI in diagnosing neonatal HIE with much lower tissue resolution; another major disadvantage is radiation exposure. For these reasons, CT is usually omitted in the diagnostic chain. However, CT is more sensitive in detecting intracranial hemorrhage than ultrasonography and requires much less time than MRI; therefore, it may be useful in some situations. ${ }^{50}$
The MRI is nowadays a standard tool for determining the pattern and severity of brain injury as well as the prognosis in infants with HIE. This method yields equal insight into all brain structures. Conventional MRI techniques have proven useful in assessing the severity of brain damage and predicting the development of newborns with HIE. ${ }^{51}$ Newer MRI techniques have also been developed that are useful in assessing the brains of newborns with HIE. Gradient-echo T2\%-weighted images and susceptibility-weighted imaging (SWI) allow for the detection of both hemorrhage and calcifications, which is important because as many as $38 \%$ of $\mathrm{TH}$-treated patients have intracranial hemorrhage $(\mathrm{ICH})$. Although preliminary results suggest that HIE remains an independent risk factor for delayed neurodevelopment in these neonates - while ICH seems to have no significant effect - further studies are needed to elucidate this. ${ }^{52}$ Diffusion-weighted imaging (DWI) has also been studied. ${ }^{53}$ Rana et al. noted that apparent diffusion coefficient value can be used as a marker to detect chronic hypoxic-ischemic brain injury. ${ }^{54}$

Magnetic resonance spectroscopy (MRS) is increasingly being performed. Mitra et al. showed that lactate/ $\mathrm{N}$-acetylaspartate levels in proton MRS within 2 weeks of birth accurately predict two-year motor, cognitive and language outcomes in neonatal encephalopathy after $\mathrm{TH} .{ }^{55}$ Lemmon et al. noted that diffusion tensor imaging (DTI) may be useful in predicting the development of newborns with HIE. ${ }^{56}$ Some authors have proposed study models and MRI estimation scales to objectify the results. Trivedi et al. proposed a validated clinical MRI injury scoring system in neonates with HIE, wherein signal abnormality was scored on T1-weighted, T2-weighted and DWI sequences, and was assessed in 5 regions; MRI injury was graded as none, mild, moderate, or severe. They showed that this scoring system is a significant predictor of neurodevelopmental outcome at 18-24 months in neonates with HIE. ${ }^{57}$ Also, Weeke et al. suggested a novel MRI score, which includes DWI and proton MRS (HMRS), assesses all important brain areas and has predictive value for outcomes at 2 years of age and at school age in infants with HIE. ${ }^{58}$

\section{Other methods}

There are several articles whose authors point out the importance of near infra-red spectroscopy in prognosis in newborns with HIE. Jain et al. found that increasing regional oxygen saturation is associated with moderate/ severe brain injury as assessed using MRI and correlates with poor neurodevelopmental outcomes in infants with HIE undergoing TH. ${ }^{59}$ Heart rate variability analysis is also drawing attention. Kayton et al. found a correlation between heart rate characteristic index score and severity of brain injury in neonates with HIE. ${ }^{60}$ Because brain damage causes a dysfunction of the thermoregulation center, the method of assessing the degree of brain damage and 
prognosis based on non-invasive estimation of endogenous heat production has also garnered interest recently. ${ }^{61}$ Preliminary results are encouraging. Mietzsch et al. examined the correlation between servo-controlled mattress temperature and the degree of brain damage in newborns treated with $\mathrm{TH}$, and concluded that the output temperature of cooling devices is a potential and easily obtainable early physiological biomarker of outcome. ${ }^{62}$

\section{Summary}

A reliable, evidence-based prognosis is essential for parental counseling regarding possible long-term sequelae. Despite the significant development of various diagnostic methods, assessing the degree of brain damage and prognosis in newborns with HIE treated with $\mathrm{TH}$ still remains a challenge, because all diagnostic tools have limitations: none are fully sensitive and specific, some cannot be performed in the intensive care unit, others are expensive, and some are only at the stage of assessing clinical suitability. The predictive value of an individual test for subsequent outcomes leaves a great deal to be desired. It seems that a combination of the prognostic tests presented in this article would provide greater prognostic accuracy for predicting long-term neurological outcomes in infants with HIE undergoing HT than any of these tests independently. ${ }^{38,45}$

Acknowledging the limitations of individual tools in certain clinical situations and integrating the information available from multiple biomarkers may help improve the accuracy of prognostication.

\section{ORCID iDs}

Wojciech Walas (1) https://orcid.org/0000-0001-7941-2718 Maria Wilińska (1) https://orcid.org/0000-0002-7557-3714 Monika Bekiesińska-Figatowska (1) https://orcid.org/0000-0003-1787-3425 Zenon Halaba (1) https://orcid.org/0000-0002-2905-0437 Robert Śmigiel (1) https://orcid.org/0000-0003-2930-9549

\section{References}

1. Graham EM, Ruis KA, Hartman AL, Northington FJ, Fox HE. A systematic review of the role of intrapartum hypoxia-ischemia in the causation of neonatal encephalopathy. Am J Obstet Gynecol. 2008;199(6): 587-595.

2. World Health Organization. http://www.childmortality.org/. Global Health Observatory (GHO); WHO 2016. Accessed April 10, 2020.

3. Jacobs SE, Berg M, Hunt R, Tarnow-Mordi WO, Inder TE, Davis PG. Cooling for newborns with hypoxic-ischaemic encephalopathy. Cochrane Syst Rev. 2013;2013(1):CD003311. https://doi.org/10.1002/14651858. CD003311.pub3

4. Wyllie J, Bruinenberg J, Roehr CC, Rüdiger M, Trevisanuto D, Urlesberger B. European Resuscitation Council Guidelines for Resuscitation 2015. Section 7. Resuscitation and support of transition of babies at birth. Resuscitation. 2015;95:249-263.

5. Laptook AR, Shankaran S, Ambalavanan N, et al; Hypothermia Subcommittee of the NICHD Neonatal Research Network. Outcome of term infants using Apgar scores at 10 minutes following hypoxic -ischemic encephalopathy. Pediatrics. 2009;124(6):1619-1626.

6. Shah P, Anvekar A, McMichael J, Rao S. Outcomes of infants with Apgar score of zero at $10 \mathrm{~min}$ : The West Australian experience. Arch Dis Child Fetal Neonatal Ed. 2015;100(6):492-494.
7. Sarnat HB, Sarnat MS. Neonatal encephalopathy following fetal distress: A clinical and encephalographic study. Arch Neurol. 1976;33(10): 696-705.

8. Grass B, Scheidegger S, Latal B, Hagmann C, Held U, Brotschi B; National Asphyxia and Cooling Register Group; Follow-up Group. Short-term neurological improvement in neonates with hypoxicischemic encephalopathy predicts neurodevelopmental outcome at 18-24 months. J Perinat Med. 2020;48(3):296-303.

9. Thompson CM, Puterman AS, Linley LL, et al. The value of a scoring system for hypoxic-ischaemic encephalopathy in predicting neurodevelopmental outcome. Acta Paediatr. 1997;86(7):757-761.

10. Mendler MR, Mendler I, Hassan MA, Mayer B, Bode H, Hummler HD. Predictive value of Thompson score for long-term neurological and cognitive outcome in term newborns with perinatal asphyxia and hypoxic-ischemic encephalopathy undergoing controlled hypothermia treatment. Neonatology. 2018;114(4):341-347.

11. Wayock CP, Meserole RL, Saria S, et al. Perinatal risk factors for severe injury in neonates treated with whole-body hypothermia for encephalopathy. Am J Obstet Gynecol. 2014;211(1):41.e1-41.e8.

12. Chiang MC, Lien R, Chu SM, et al. Serum lactate, brain magnetic resonance imaging and outcome of neonatal hypoxic-ischemic encephalopathy after therapeutic hypothermia. Pediatr Neonatol. 2016;57(1): 35-40.

13. Malin GL, Morris RK, Khan KS. Strength of association between umbilical cord $\mathrm{pH}$ and perinatal and long term outcomes: Systematic review and meta-analysis. BMJ. 2010;340:c1471.

14. Muniraman H, Gardner D, Skinner J, et al. Biomarkers of hepatic injury and function in neonatal hypoxic-ischemic encephalopathy and with therapeutic hypothermia. Eur J Pediatr. 2017;176(10):1295-1303.

15. Montaldo P, Rosso R, Chello G, Giliberti P. Cardiac troponin I concentrations as a marker of neurodevelopmental outcome at 18 months in newborns with perinatal asphyxia. J Perinatol. 2014;34(4):292-295.

16. Hong F, Song L, Zhu YY, Ji JH, Zhu MJ, Xu M. Cardiac troponin I, myoglobin, and creatine kinase-Mb as new biomarkers for diagnosis of neonatal hypoxic-ischemic encephalopathy. J Biol Regul Homeost Agents. 2019;33(4):1201-1207.

17. Yum SK, Moon CJ, Youn YA, Sung IK. Changes in lactate dehydrogenase are associated with central gray matter lesions in newborns with hypoxic-ischemic encephalopathy. JMatern Fetal Neonatal Med. 2017;30(10):1177-1181.

18. Chaparro-Huerta V, Flores-Soto ME, Merin Sigala ME, et al. Proinflammatory cytokines, enolase and S-100 as early biochemical indicators of hypoxic-ischemic encephalopathy following perinatal asphyxia in newborns. Pediatr Neonatol. 2017;58(1):70-76.

19. Roka A, Kelen D, Halasz J, Beko G, Azzopardi D, Szabo M. Serum S100B and neuron-specific enolase levels in normothermic and hypothermic infants after perinatal asphyxia. Acta Paediatr. 2012;101(3):319-323.

20. Giuseppe D, Sergio C, Pasqua B, et al. Perinatal asphyxia in preterm neonates leads to serum changes in protein S-100 and neuron specific enolase. Curr Neurovasc Res. 2009;6(2):110-116.

21. Massaro AN, Chang T, Baumgart S, McCarter R, Nelson KB, Glass P. Biomarkers $\mathrm{S} 100 \mathrm{~B}$ and neuron-specific enolase predict outcome in hypothermia-treated encephalopathic newborns. Pediatr Crit Care Med. 2014;15(7):615-622.

22. Ennen CS, Huisman TA, Savage WJ, et al. Glial fibrillary acidic protein as a biomarker for neonatal hypoxic-ischemic encephalopathy treated with whole-body cooling. Am J Obstet Gynecol. 2011;205(3):251.e1-e7.

23. Florio P,Frigiola $A$, Battista R, et al. Activin A in asphyxiated full-term newborns with hypoxic-ischemic encephalopathy. Front Biosci (Elite Ed). 2010;2:36-42.

24. Douglas-Escobar MV, Heaton SC, Bennett J, et al. UCH-L1 and GFAP serum levels in neonates with hypoxic-ischemic encephalopathy: A single center pilot study. Front Neurol. 2014;5:273.

25. Lv HY, Wu SJ, Gu XL, et al. Predictive value of neurodevelopmental outcome and serum tau protein level in neonates with hypoxic-ischemic encephalopathy. Clin Lab. 2017;63(7):1153-1162.

26. Shah DK, Ponnusamy V, Evanson J, et al. Raised plasma neurofilament light protein levels are associated with abnormal MRI outcomes in newborns undergoing therapeutic hypothermia. Front Neurol. 2018;9:86.

27. Chalak LF, Sánchez PJ, Adams-Huet B, Laptook AR, Heyne RJ, Rosenfeld CR. Biomarkers for severity of neonatal hypoxic-ischemic encephalopathy and outcomes in newborns receiving hypothermia therapy. J Pediatr. 2014;164(3):468-474. 
28. Graham EM, Everett AD, Delpech JC, Northington FJ. Blood biomarkers for evaluation of perinatal encephalopathy: State of the art. Curr Opin Pediatr. 2018;30(2):199-203.

29. Li Y, Dammer EB, Zhang-Brotzge X, et al. Osteopontin is a blood biomarker for microglial activation and brain injury in experimental hypoxic-ischemic encephalopathy. eNeuro. 2017;4(1):ENEURO.025316.2016. doi:10.1523/ENEURO.0253-16.2016

30. Aly H, Hassanein S, Nada A, Mohamed MH, Atef SH, Atiea W. Vascular endothelial growth factor in neonates with perinatal asphyxia. Brain Dev. 2009;31(8):600-604.

31. Hagag AA, El Frargy MS, Abd El-Latif AE. Study of cord blood erythropoietin, leptin and adiponectin levels in neonates with hypoxicischemic encephalopathy. Endocr Metab Immune Disord Drug Targets. 2020;20(2):213-220.

32. Ahearne CE, Denihan NM, Walsh BH, et al. Early cord metabolite index and outcome in perinatal asphyxia and hypoxic-ischaemic encephalopathy. Neonatology. 2016;110(4):296-302.

33. Denihan NM, Kirwan JA, Walsh BH, et al. Untargeted metabolomic analysis and pathway discovery in perinatal asphyxia and hypoxic-ischaemic encephalopathy. J Cereb Blood Flow Metab. 2019;39(1): 147-162.

34. Liu JF, Wu HW, Li ZG, Lu GZ, Yang X. aEEG monitoring analysis of lesion degree and long-term prognosis in newborns with HIE. Eur Rev Med Pharmacol Sci. 2016;20(13):2863-2867.

35. Sewell EK, Vezina G, Chang T, et al. Evolution of amplitude-integrated electroencephalogram as a predictor of outcome in term encephalopathic neonates receiving therapeutic hypothermia. Am J Perinatol. 2018;35(3):277-285

36. Goeral K, Urlesberger B, Giordano V, et al. Prediction of outcome in neonates with hypoxic-ischemic encephalopathy II: Role of amplitude-integrated electroencephalography and cerebral oxygen saturation measured by near-infrared spectroscopy. Neonatology. 2017; 112(3):193-202.

37. Skranes JH, Løhaugen G, Schumacher EM, et al. Amplitude-integrated electroencephalography improves the identification of infants with encephalopathy for therapeutic hypothermia and predicts neurodevelopmental outcomes at 2 years of age. J Pediatr. 2017;187: 34-42.

38. Del Río R, Ochoa C, Alarcon A, Arnáez J, Blanco D, García-Alix A. Amplitude integrated electroencephalogram as a prognostic tool in neonates with hypoxic-ischemic encephalopathy: A systematic review. PLoS One. 2016;11(11):e0165744.

39. Chandrasekaran M, Chaban B, Montaldo P, Thayyil S. Predictive value of amplitude-integrated EEG (aEEG) after rescue hypothermic neuroprotection for hypoxic-ischemic encephalopathy: A meta-analysis. J Perinatol. 2017;37(6):684-689.

40. Shah DK, Wusthoff CJ, Clarke $P$, et al. Electrographic seizures are associated with brain injury in newborns undergoing therapeutic hypothermia. Arch Dis Child Fetal Neonatal Ed. 2014;99(3):219-224.

41. Awal MA, Lai MM, Azemi G, Boashash B, Colditz PB. EEG background features that predict outcome in term neonates with hypoxic-ischaemic encephalopathy: A structured review. Clin Neurophysiol. 2016; 127(1):285-296.

42. Dunne JM, Wertheim D, Clarke P, et al. Automated electroencephalographic discontinuity in cooled newborns predicts cerebral MR and neurodevelopmental outcome. Arch Dis Child Fetal Neonatal Ed. 2017;102(1):58-64.

43. Weeke LC, Boylan GB, Pressler RM, et al. NEonatal seizure treatment with Medication Off-patent (NEMO) consortium. Role of EEG background activity, seizure burden and MRI in predicting neurodevelopmental outcome in full-term infants with hypoxic-ischaemic encephalopathy in the era of therapeutic hypothermia. Eur J Paediatr Neurol. 2016;20(6):855-864.

44. Han Y, Fu N, Chen W, et al. Prognostic value of electroencephalography in hypothermia-treated neonates with hypoxic-ischemic encephalopathy: A meta-analysis. Pediatr Neurol. 2019;93:3-10.
45. Liu W, Yang Q, Wei H, Dong W, Fan Y, Hua Z. Prognostic value of clinical tests in neonates with hypoxic-ischemic encephalopathy treated with therapeutic hypothermia: A systematic review and meta-analysis. Front Neurol. 2020;11:133.

46. Suppiej A, Cappellari A, Talenti G, et al. Bilateral loss of cortical SEPs predicts severe MRI lesions in neonatal hypoxic-ischemic encephalopathy treated with hypothermia. Clin Neurophysiol. 2018;129(1):95-100.

47. Cainelli E, Trevisanuto D, Cavallin F, Manara R, Suppiej A. Evoked potentials predict psychomotor development in neonates with normal MRI after hypothermia for hypoxic-ischemic encephalopathy. Clin Neurophysiol. 2018;129(6):1300-1306.

48. Gerner GJ, Burton VJ, Poretti A, et al. Transfontanellar duplex brain ultrasonography resistive indices as a prognostic tool in neonatal hypoxic-ischemic encephalopathy before and after treatment with therapeutic hypothermia. J Perinatol. 2016;36(3):202-206.

49. Annink KV, de Vries LS, Groenendaal F, et al. The development and validation of a cerebral ultrasound scoring system for infants with hypoxic-ischaemic encephalopathy. Pediatr Res. 2020;87(Suppl 1): 59-66.

50. Zhang Y, Zhang JL, Li Y. Computed tomography diagnosis of neonatal hypoxic-ischemic encephalopathy combined with intracranial hemorrhage and clinical nursing treatment. J Biol Regul Homeost Agents. 2016;30(2):511-515.

51. Thayyil S, Chandrasekaran M, Taylor A, et al. Cerebral magnetic resonance biomarkers in neonatal encephalopathy: A meta-analysis. Pediatrics. 2010;125(2):382-395.

52. Lakatos A, Kolossváry M, Szabó M, et al. Neurodevelopmental effect of intracranial hemorrhage observed in hypoxic-ischemic brain injury in hypothermia-treated asphyxiated neonates: An MRI study. NCBINCBI Logo BMC Pediatr. 2019;19(1):430.

53. Bekiesinska-Figatowska M, Duczkowska A, Szkudlinska-Pawlak S, et al. Diffusion restriction in the corticospinal tracts and the corpus callosum in neonates after cerebral insult. Brain Dev. 2017;39(3):203-210.

54. Rana L, Sood D, Chauhan R, et al. MR imaging of hypoxic-ischemic encephalopathy: Distribution patterns and ADC value correlations. Eur J Radiol Open. 2018;5:215-220.

55. Mitra S, Kendall GS, Bainbridge A, et al. Proton magnetic resonance spectroscopy lactate/ $\mathrm{N}$-acetylaspartate within 2 weeks of birth accurately predicts 2 -year motor, cognitive and language outcomes in neonatal encephalopathy after therapeutic hypothermia. Arch Dis Child Fetal Neonatal Ed. 2019;104(4):424-432.

56. Lemmon ME, Wagner MW, Bosemani T, et al. Diffusion tensor imaging detects occult cerebellar injury in severe neonatal hypoxicischemic encephalopathy. Dev Neurosci. 2017;39(1-4):207-214.

57. Trivedi SB, Vesoulis ZA, Rao R, et al. A validated clinical MRI injury scoring system in neonatal hypoxic-ischemic encephalopathy. Pediatr Radiol. 2017;47(11):1491-1499.

58. Weeke LC, Groenendaal F, Mudigonda K, et al. A novel magnetic resonance imaging score predicts neurodevelopmental outcome after perinatal asphyxia and therapeutic hypothermia. J Pediatr. 2018;192: 33-40.e2.

59. Jain SV, Pagano L, Gillam-Krakauer M, Slaughter JC, Pruthi S, Engelhardt B. Cerebral regional oxygen saturation trends in infants with hypoxic-ischemic encephalopathy. Early Hum Dev. 2017;113:55-61.

60. Kayton A, DeGrazia M, Sharpe E, Smith D, Perez JA, Weiss MD. Correlation between heart rate characteristic index score and severity of brain injury in neonates with hypoxic-ischemic encephalopathy. Adv Neonatal Care. 2020;20(4):E70-E82. doi:10.1097/ANC.00000 00000000686

61. Gonzaga e Silva ABC, Laszczyk J, Wrobel LC, Ribeiro FLC, Nowak AJ. A thermoregulation model for hypothermic treatment of neonates. Med Eng Phys. 2016;38(9):988-998.

62. Mietzsch U, Radhakrishnan R, Boyle FA, Juul S, Wood TR. Active cooling temperature required to achieve therapeutic hypothermia correlates with short-term outcome in neonatal hypoxic-ischaemic encephalopathy. J Physiol. 2020;598(2):415-424 